\title{
Effect of Phenobarbital on the Pharmacokinetics of Rutaecarpine and its Metabolite in Rats
}

\author{
Ju Hyun Kim, Sang Kyu Lee, Young Min Seo, Jae Ho Chol, Sil Shin, Mi Jeong Kang, \\ Dong Hyeon KIm, Hye Gwang Jeong ${ }^{1}$, Yurngdong JAHNG, and Tae Cheon JeOnG* \\ College of Pharmacy, Yeungnam University, Gyeongsan 712-749, Korea \\ ${ }^{1}$ College of Pharmacy, Chosun University, Gwangju 501-759, Korea
}

(Received July 15, 2008; Revised July 25, 2008; Accepted August 27, 2008)

\begin{abstract}
To investigate the possible interaction between rutaecarpine and phenobarbital in rats, phenobarbital in saline at $80 \mathrm{mg} / \mathrm{kg}$ was given ip to male SD rats for 3 consecutive days. Saline was given to control animals. One day after phenobarbital pre-treatment, rutaecarpine at $16 \mathrm{mg} / \mathrm{kg}$ was administered through penile vein. Blood was collected and analyzed by using HPLC. The pharmacokinetic parameters were determined with the non-compartmental model. Pre-treatment with phenobarbital significantly altered the pharmacokinetic profiles of rutaecarpine and its metabolite, 10-hydroxyrutaecarpine. The AUC of rutaecarpine was reduced to approximately $50 \%$ of control and the plasma half-life of rutaecarpine was significantly shortened when compared with control. In addition, the Cmax of 10-hydroxyrutaecarpine was increased approximately $160 \%$ of control. The AUC and the plasma half-life of 10-hydroxyrutaecarpine were decreased to $76.9 \%$ of control and to $82.7 \mathrm{~min}$ from $175.9 \mathrm{~min}$, respectively. The results suggested that phenobarbital might accelerate the metabolism of rutaecarpine, thereby changing the pharmacokinetic parameters of rutaecarpine in male SD rats.
\end{abstract}

Key words: Rutaecarpine, Phenobarbital, Drug interaction, Pharmacokinetics, Metabolite, in vivo

\section{INTRODUCTION}

Rutaecarpine [8,13-dihydro-7H-indolo-(2',3':3,4)-pyrido(2,1-b)-quinazolin-5-one] is an alkaloid originally isolated from the unripe fruit of Evodia rutaecarpa (Chiou et al., 1996). In addition to its traditional use in treatment of gastrointestinal disorders, rutaecarpine has recently been characterized to have an anti-inflammatory activity through cyclooxygenase-2 inhibition (Moon et al., 1999; Woo et al., 2001). More recently, in order to develop rutaecarpine as an anti-inflammatory agent, a simple and practical method for the synthesis of rutaecarpine in a large quantity was successfully established by our group (Lee et al., 2001). Previous studies reported that cytochrome P450 (CYP) 1A and 2B might predominantly metabolize rutaecarpine in rat liver microsomes (Lee et al., 2004a) and that nine phase I metabolites of rutaecarpine were identified by using liquid chromatographyelectrospray ionization tandem mass spectrometry (Lee

\footnotetext{
${ }^{*}$ Corresponding author

Tel: +82-53-810-2819, Fax: +82-53-810-4654

E-mail: taecheon@yumail.ac.kr
}

et al., 2004b).

Phenobarbital (PB), one of the antiepileptic drugs, induces drug-metabolizing enzymes in laboratory animals and human, including CYP 2B and 3A (Park et al., 2005). PB has been widely used as a prototype inducer for drug metabolism in pharmacological and toxicological investigations.

Although many constituents isolated from natural resources have been investigated for the development of new drug candidates, the possible herb-drug interactions were hardly studied. Particularly, the in vivo interaction of rutaecarpine with CYP inducers and inhibitors has not been investigated to date. To develop rutaecarpine as an anti-inflammatory drug, our group has focused our research on its metabolism (Lee et al., 2004a, Lee et al., 2005, Park et al., 2005) and, for this reason, it was necessarily required to study the possible interaction of rutaecarpine with a well known CYP inducer, PB.

In the present study, the pharmacokinetic parameters of rutaecarpine and its metabolite were investigated in rats following pre-treatment with phenobarbital as a part of new drug development. 


\section{MATERIALS AND METHODS}

\section{Materials}

Rutaecarpine (purity, >99\%) used in this study was chemically synthesized in our group (Lee et al., 2001). Tryptanthrin (purity, $>99.8 \%$ ) was obtained by the chemical synthesis described previously (Son et al., 2003). PB was obtained from Sigma Chemical Co. (St. Louis, MO, USA). All other chemicals were of analytical grade and all solvents were of HPLC grade.

\section{Animal studies}

Specific pathogen-free male Sprague Dawley rats (250$280 \mathrm{~g}$ ) were obtained from the Orient Co. (Seoul, Korea). The animals received at 6 weeks of age were acclimated for at least 1 week. Upon arrival, animals were randomized and housed 3 per cage. The animal quarters were strictly maintained at $23 \pm 3^{\circ} \mathrm{C}$ and $50 \pm 10 \%$ relative humidity. A $12 \mathrm{~h}$ light and dark cycle was used with an intensity of 150 - 300 Lux. All animal procedures were followed based on a guideline recommended by the Society of Toxicology (USA) in 1989. PB in saline at $80 \mathrm{mg} / \mathrm{kg}$ was given ip to rats for 3 consecutive days. For control, saline was given to rats at $10 \mathrm{ml} / \mathrm{kg}$. $24 \mathrm{~h}$ after the last dosing, rutaecarpine in $1 \%$ povidone solution at $16 \mathrm{mg} / \mathrm{kg}$ was administered into penile vein. Approximately $300 \mu \mathrm{l}$ of blood samples were collected from the subclavian vein immediately before and $5,10,20,40,60,120,180$, and $240 \mathrm{~min}$ after the rutaecarpine administration. Blood samples were centrifuged at $3000 \times \mathrm{g}$ for $15 \mathrm{~min}$ at $4^{\circ} \mathrm{C}$ to obtain sera.

\section{HPLC analysis}

$5 \mu \mathrm{l}$ of internal standard (IS; $5 \mu \mathrm{g} / \mathrm{ml}$ of tryptanthrin) was added to serum samples and then $1 \mathrm{ml}$ of ethyl acetate was added to deproteinize serum samples. Following centrifugation at $3,000 \times \mathrm{g}$ for $10 \mathrm{~min}$ at $15^{\circ} \mathrm{C}$, the supernatant $(0.85 \mathrm{ml})$ was completely evaporated with a stream of nitrogen gas. The residue was reconstituted with $100 \mu \mathrm{l}$ of methanol and centrifuged at $10,000 \times \mathrm{g}$ for $10 \mathrm{~min} .20 \mu \mathrm{l}$ aliquot was injected into the HPLC system.

The HPLC system consisted of a pump (LC-20AD), an autoinjector (SIL-20A), a UV-Visible detector (SPD-20A), and a communications bus module (CBM-20A) from Shimadzu Scientific Instruments (Kyoto, Japan). A Waters COSMOSIL ${ }_{5} \mathrm{C}_{18}$-MS-II column $(5 \mu \mathrm{m}, 4.6 \times 250 \mathrm{~mm})$ was used for the HPLC separation.

Separation was conducted using a gradient solvent system from $40: 60$ to $80: 20$ for 35 min with $100 \%$ of acetonitrile:20 mM ammonium formate buffer, $\mathrm{pH} 6.8$, at a flow rate of $1.2 \mathrm{ml} / \mathrm{min}$. The eluate was monitored at 344 $\mathrm{nm}$. The data was acquired as the ratio of the AUC of samples with AUC of IS. The spiked concentration of the calibration standard was linear over the concentration range of $0.05 \mathrm{mg} / \mathrm{ml}$ to $3 \mathrm{mg} / \mathrm{ml}$.

\section{Pharmacokinetic parameters and statistics}

The pharmacokinetic parameters were determined using the standard non-compartmental method. Serum AUC was calculated using WinNonlin (version 1.1, Scientific Consulting, KY, USA) with a log linear trapezoidal method. The results were expressed as mean $\pm S$.E. and the significance of data was analyzed with Student's $t$-test. The values significantly different from control were expressed as asterisks at either $P<0.05\left(^{*}\right)$ or $\left.P<0.01{ }^{* *}\right)$.

\section{RESULTS}

Representative UV chromatograms of blank serum and serum isolated from rats treated intravenously with rutaecarpine are shown in Figure 1. Rutaecarpine, IS, and 10hydroxyrutaecarpine were detected at the retention time of
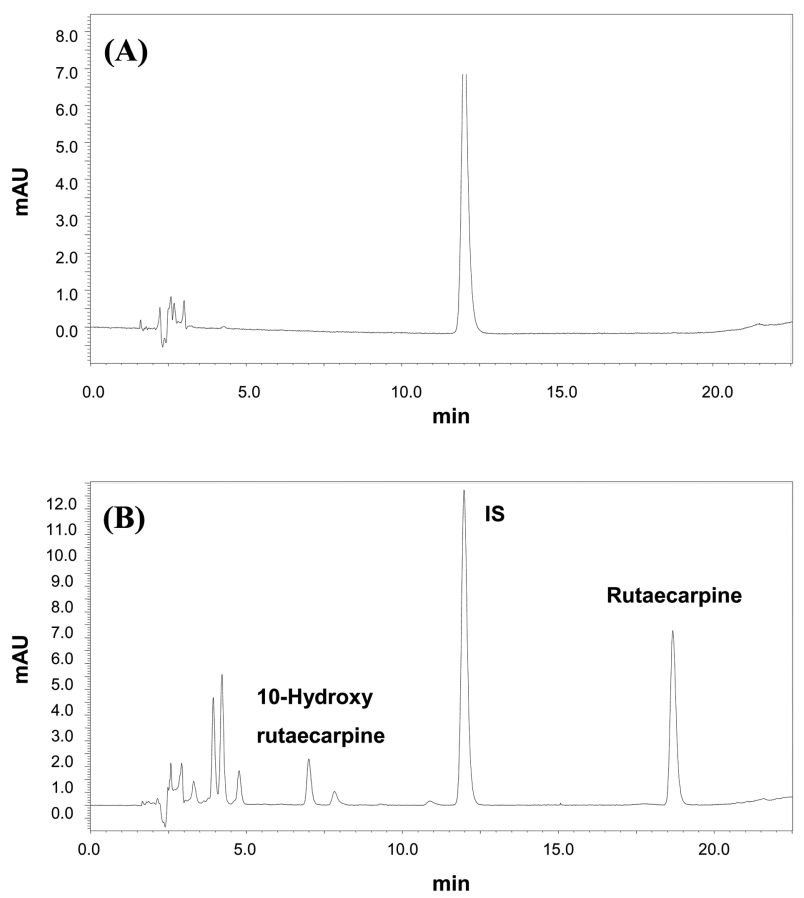

Fig. 1. Chromatograms of the blank serum (A) and the serum treated intravenously with $16 \mathrm{mg} / \mathrm{kg}$ rutaecarpine for $10 \mathrm{~min}$ (B). Rutaecarpine, internal standard (IS; tryptanthrin) and 10hydroxyrutaecarpine were detected at the retention times of $18.6,11.9$, and $6.9 \mathrm{~min}$, respectively. The concentration of IS, tryptanthrin, was $5 \mu \mathrm{g} / \mathrm{ml}$. 
Table I. Pharmacokinetic parameters of rutaecarpine and its metabolite following phenobarbital pre-treatment in rats

\begin{tabular}{|c|c|c|c|c|c|}
\hline \multirow[b]{2}{*}{ Parameters } & \multicolumn{2}{|c|}{ Rutaecarpine } & \multirow[b]{2}{*}{ Parameters } & \multicolumn{2}{|c|}{ 10-Hydroxyrutaecarpine } \\
\hline & Control & $\begin{array}{c}\text { Phenobarbital } \\
\text { pre-treated }\end{array}$ & & Control & $\begin{array}{c}\text { Phenobarbital } \\
\text { pre-treated }\end{array}$ \\
\hline- & - & - & $\mathrm{T}_{\max }(\min )$ & $26.7 \pm 6.7$ & $10.0 \pm 0.0$ \\
\hline- & - & - & $\mathrm{C}_{\max }(\%)$ & $100.0 \pm 0.0$ & $160.0 \pm 27.4^{*}$ \\
\hline $\mathrm{AUC}_{\infty}(\mu \mathrm{g} \cdot \mathrm{min} / \mathrm{ml})$ & $115.7 \pm 8.8$ & $64.0 \pm 0.3^{*}$ & AUC (\%) & $100.0 \pm 0.0$ & $76.9 \pm 2.6^{*}$ \\
\hline $\mathrm{T}_{1 / 2}(\min )$ & $56.7 \pm 1.2$ & $35.7 \pm 0.8^{\star *}$ & $\mathrm{~T}_{1 / 2}(\min )$ & $175.9 \pm 2.7$ & $82.7 \pm 10.1^{\text {** }}$ \\
\hline
\end{tabular}

Phenobarbital in saline at $80 \mathrm{mg} / \mathrm{kg}$ was given ip to male SD rats for 3 consecutive days. For control, saline was given to rats at $10 \mathrm{ml} / \mathrm{kg} .24 \mathrm{~h}$ after the last dosing, rutaecarpine in $1 \%$ povidone solution at $16 \mathrm{mg} / \mathrm{kg}$ was administered through penile vein. Blood samples were collected from the subclavian vein immediately before and 5, 10, 20, 40,60, 120, 180, and 240 min after the rutaecarpine administration. Each value represents mean \pm S.E. of three animals. The asterisks indicate the values significantly different from control at either $P<0.05\left(^{*}\right)$ or $P<0.01\left(^{* *}\right)$.

18.6, 11.9, and $6.9 \mathrm{~min}$, respectively. 10-Hydroxyrutaecarpine was identified and confirmed by using liquid chromatography-electrospray ionization tandem mass spectrometry and by previous literatures (Lee et al., 2005, Ueng et al., 2005).

The mean serum concentration-time curve of rutaecarpine and 10-hydroxyrutaecarpine are shown in Figure 2 and Figure 3, respectively. As shown in Table I, the pharmacokinetic parameters of the group pre-treated with PB were significantly changed when compared with the control where rutaecarpine was administered alone. The AUC and the terminal plasma half-life $\left(T_{1 / 2}\right)$ of rutaecarpine decreased significantly in the group pre-treated with PB.

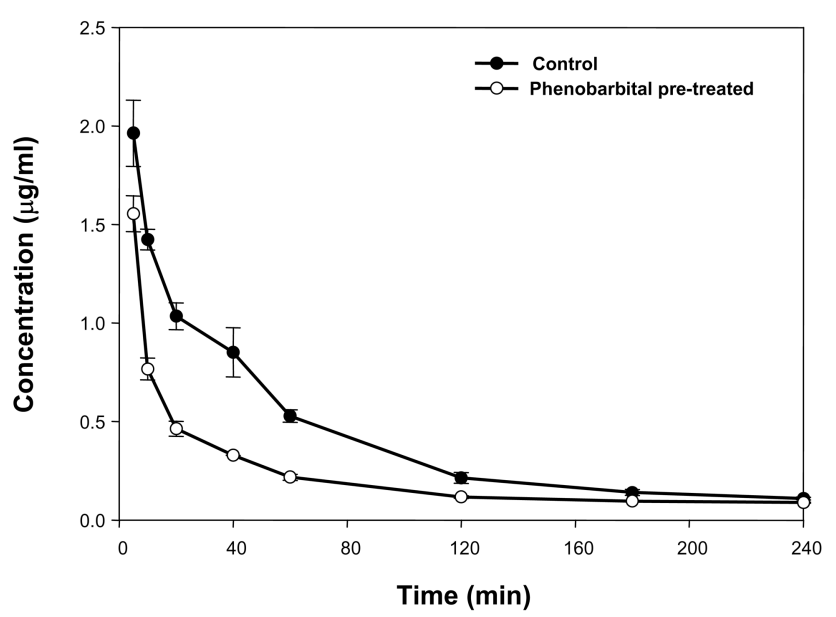

Fig. 2. Mean serum concentration-time profile of rutaecarpine in male SD rats following an intravenous injection with $16 \mathrm{mg} /$ $\mathrm{kg}$ rutaecarpine. Blood was collected from the subclavian vein immediately before and 5, 10, 20, 40, 60, 120, 180, and 240 min after the dose. Each value represents the mean \pm S.E. of three animals.
Although the authentic standard of 10-hydroxyrutaecarpine was not available, some pharmacokinetic parameters could be deduced in the present study. When rats were pre-treated with $\mathrm{PB}$, the Cmax of 10-hydroxyrutaecarpine was increased to 1.6-fold of control and the $T_{\max }$ reached approximately 2.7 -fold faster than control group. In addition, PB pretreatment significantly decreased the AUC of metabolite to $76.9 \%$ of control, and the terminal plasma half-life $\left(T_{1 / 2}\right)$ to $82.7 \mathrm{~min}$ from $175.9 \mathrm{~min}$ of control.

\section{DISCUSSION}

There are many reasons causing failure in new drug

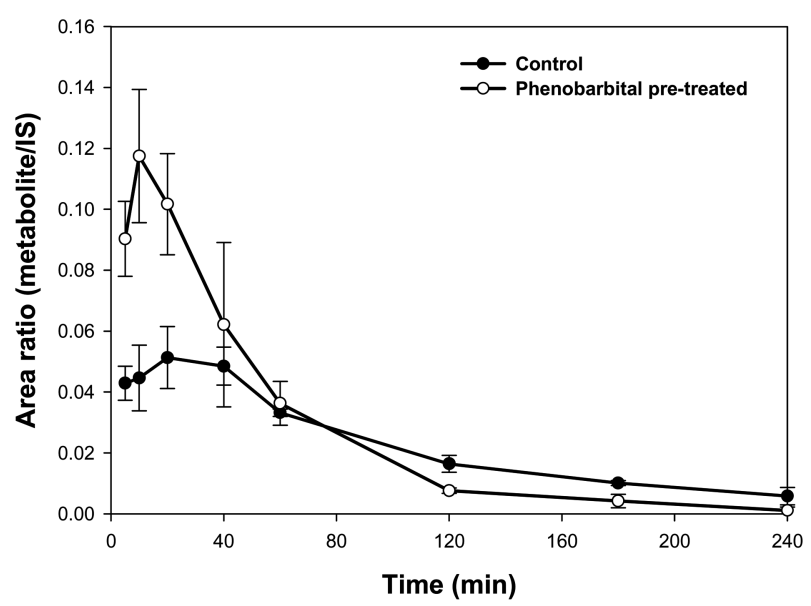

Fig. 3. Mean serum area ratio for metabolites/internal standard (IS)-time profile of 10-hydroxyrutaecarpine in male SD rats following an intravenous injection with $16 \mathrm{mg} / \mathrm{kg}$ rutaecarpine. Blood was collected from the subclavian vein immediately before and $5,10,20,40,60,120,180$, and $240 \mathrm{~min}$ after the dose. Each value represents the mean \pm S.E. of three animals. 
development, such as inappropriateness of drug metabolic or pharmacokinetic characteristics including decreased pharmacological effect in in vivo system, toxicity, very short half-life, and induction of specific enzymes. To overcome or avoid these problems, the importance of studies on drug metabolism and pharmacokinetics is emphasized in the early stage of drug development nowadays. In addition, studying pharmacokinetic profiles of parent compound together with their metabolites can play a very useful role in new drug development to prevent possible financial damage that might be occurred from the failure in a late stage. For these reasons, the possible interaction of rutaecarpine with $\mathrm{PB}$ was investigated in the present study. PB was selected as a CYP inducer, because our previous report indicated that microsomes isolated from PB-pretreated rats could significantly increase the metabolism of rutaecarpine in vitro (Lee et al., 2004a).

As mentioned in the Introduction, rutaecarpine has beneficial pharmacological activities including anti-inflammatory activities (Moon et al., 1999, Woo et al., 2001). In addition, we have recently identified 9 phase I metabolites with their chemical structures by using tandem mass spectrometry (Lee et al., 2004b). Therefore, rutaecarpine would be a good model compound to study the drug-drug interaction, because one can study the possible kinetic changes in metabolite production together with the parent drug.

In the present LC conditions, however, 10-hydroxyrutaecarpine was only detected among 9 phase I metabolites in rats. Nevertheless, we could deduce some pharmacokinetic changes of rutaecarpine and its 10-hydroxy metabolite following PB pretreatment. Pretreatment of rats with PB significantly reduced the plasma level of rutaecarpine with its half-life. In addition, PB pretreatment significantly changed the $T_{\max }$ of 10-hydroxy metabolite. These results clearly indicated that the induction of CYP by PB significantly increased in the metabolism of rutaecarpine to its phase I metabolite. The present results were consistent with our previous reports that the metabolism of rutaecarpine is CYP dependent in rat liver microsomes (Lee et al., 2004a, Lee et al., 2004b). Although only one metabolite was detected in the present study, it was evident that $\mathrm{PB}$ can modulate the metabolism of rutaecarpine in rats. It would be more informative when the pharmacokinetic parameters for all phase I metabolites are available.

\section{ACKNOWLEDGEMENT}

This work was supported by the grants from Korea Research Foundation (KRF-2006-E00154).

\section{REFERENCES}

Chiou, W.F., Liao, J.P., and Chen, C.F. (1996) Comparative study of the vasodilatory effects of three quinazoline alkaloids isolated from Evodia rutaecarpa. J. Nat. Prod. 59, 374378.

Lee, S.H., Kim, S.I., Park, J.G., Lee, E.S., and Jahng, Y. (2001) A simple synthesis of rutaecarpine. Heterocycles 55, 15551559.

Lee, S.K., Kim, N.H., Lee, J., Kim, D.H., Lee, E.S., Choi, H.G., Chang, H.W., Jahng, Y., and Jeong, T.C. (2004a) Induction of cytochrome P450s by rutaecarpine and metabolism of rutaecarpine by cytochrome P450s. Planta Med. 70, 753-757.

Lee, S.K., Lee, D.W., Jeon, T.W., Jin, C.H., Kim, G.H., Jun, I.H., Lee, D.J., Kim, S.I., Kim, D.H., Jahng, Y., and Jeong, T.C. (2005) Characterization of the phase 2 metabolites of rutaecarpine in the rat by liquid chromatography-electrospray ionization tandem mass spectrometry. Xenobiotica 35, 11351145.

Lee, S.K., Lee, J., Lee, E.S., Jahng, Y., Kim, D.H., and Jeong, T.C. (2004b) Characterization of in vitro metabolites of rutaecarpine in rat liver microsomes using liquid chromatography/ tandem mass spectrometry. Rapid Commun. Mass Spectrom. 18, 1073-1080.

Moon, T.C., Murakami, M., Kudo, I., Son, K.H., Kang, S.S., and Chang, H.W. (1999) A new class of COX-2 inhibitor, rutaecarpine from Evodia rutaecarpa. Inflamm. Res. 48, 621-625.

Park, E.J., Cho, H.Y., and Lee, Y.B. (2005) Effect of cimetidine and phenobarbital on metabolite kinetics of omeprazole in rats. Arch. Pharm. Res. 28, 1196-1202.

Son, J.K., Park, J.G, and Jahng, Y. (2003) A simple synthesis of tryptanthrin. Heterocycl. Commun. 9, 621-624.

Ueng, Y.F., Yu, H.J., Lee, C.H., Peng, C., Jan, W.C., Ho, L.K., Chen, C.F., and Don, M.J. (2005) Identification of the microsomal oxidation metabolites of rutaecarpine, a main active alkaloid of the medicinal herb Evodia rutaecarpa. J. Chromatogr. A 1076, 103-109.

Woo, H.G. Lee, C.H., Noh, M.S., Lee, J.J., Jung, Y.S., Baik, E.J., Moon, C.H., and Lee, S.H. (2001) Rutaecarpine, a quinazolinocarboline alkaloid, inhibits prostaglandin production in RAW264.7 macrophages. Planta Med. 67, 505-509. 